Pacific Journal of Mathematics

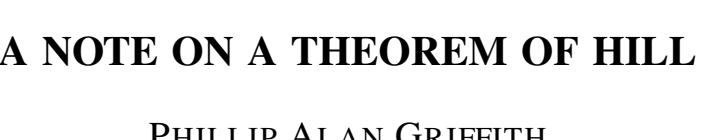




\section{A NOTE ON A THEOREM OF HILL}

\section{PHILLIP GRIFFITH}

Recently Hill has shown the existence of an abelian $p$ group with the property that each infinite subgroup can be embedded in a direct summand of the same cardinality but the group is not a direct sum of countable groups. Megibben has since observed that this phenomenon occurs in a larger class of abelian groups. In this note we show that such pathology is present in modules for a rather wide class of rings. In fact, the lack of such phenomena for a particular class of modules serves as a characterization for left perfect rings. Our results also yield some facts concerning pure injective modules.

All rings in this paper are associative with identity and all modules are unital.

2. A characterization of left perfect rings. Bass [1] calls a ring $R$ left perfect if each left $R$-module has a projective cover (projective cover is the dual of injective envelope). Among several other characterizations of left perfect rings, Bass proves that $R$ is left perfect if and only if $R$ has the descending chain condition on principal right ideals. Hence, assuming that $R$ is not left perfect, we can obtain a strictly decreasing sequence of principal right ideals of the form

$$
a_{1} R \supset a_{1} a_{2} R \supset \cdots \supset a_{1} \cdots a_{n} R \supset \cdots .
$$

We set $P=\Pi_{n<\omega} R e_{n}$, where $R e_{n} \cong R$ for each $n$, and we denote by $S$ the submodule of finitely nonzero sequences in $P$. We shall use the notation $\sum_{i=m}^{n} r_{i} e_{i}$, for $m \leqq n$, to denote a vector in $P$ whose $i$ th coordinate is zero for $i>n$ and $i<m$ and whose $i$ th coordinate is $r_{i} e_{i}$ for $m \leqq i \leqq n$. We define elements

$$
c^{(m)}=\sum_{i \geq m}\left(a_{m} \cdots a_{i}\right) e_{i} \in P \quad \text { for } \quad m=1,2, \cdots .
$$

Let $A$ be the submodule of $P$ generated by $S$ and the elements $c^{(m)}$ for $m=1,2, \cdots$. With this notation established, we prove the following lemma.

Lemma 2.1. Let $R$ be a ring that is not left perfect and let $A$ and $S$ be defined as above. Then $A$ is free and $S$ is not a direct summand of $A$.

Proof. First we note that if $n<m$, then 


$$
c^{(n)}=\sum_{i \geqq n}^{m-1}\left(a_{n} \cdots a_{i}\right) e_{i}+\left(a_{n} \cdots a_{m-1}\right) c^{(m)}
$$

and in particular

$$
c^{(n)}=a_{n}\left(e_{n}+c^{(n+1)}\right) \quad \text { for } \quad n=1,2, \cdots \text {. }
$$

Now suppose that $A=S \oplus B$. Then $c^{(n)}=s_{n}+b_{n}$ where $s_{n} \in S$ and $b_{n} \neq 0 \in B$. From property (*) above, we have that, for $n>1$,

$$
c^{(1)}=s_{1 n}+\left(a_{1} \cdots a_{n-1}\right) c^{(n)} \quad \text { where } \quad s_{1 n} \in S \text {. }
$$

Therefore

$$
\begin{aligned}
s_{1}+b_{1} & =c^{(1)}=s_{1 n}+\left(a_{1} \cdots a_{n-1}\right) c^{(n)} \\
& =s_{1 n}+\left(a_{1} \cdots a_{n-1}\right)\left(s_{n}+b_{n}\right) \\
& =s_{1 n}+\left(a_{1} \cdots a_{n-1}\right) s_{n}+\left(a_{1} \cdots a_{n-1}\right) b_{n} .
\end{aligned}
$$

Hence $s_{1}=s_{1 n}+\left(a_{1} \cdots a_{n-1}\right) s_{n}$ and $b_{1}=\left(a_{1} \cdots a_{n-1}\right) b_{n}$ for each $n>1$. Therefore $c^{(1)}=s_{1}+\left(a_{1} \cdots a_{n-1}\right) b_{n}$ for $n=2,3, \cdots$. Since $s_{1}$ has only finitely many nonzero coordinates, it follows that there is a positive integer $r$ such that $a_{1} \cdots a_{r}=a_{1} \cdots a_{r} a_{r+1} y$. But this implies that $a_{1} \cdots a_{r} R=a_{1} \cdots a_{r+1} R$ which is a contradiction. Thus $S$ is not a summand of $A$.

To show that $A$ is free, let $y_{n}=e_{n}+c^{(n+1)}$ for $n=1,2, \cdots$. Since $c^{(n)}=a_{n} y_{n}$ by property $(*)$ above, it follows that $A$ is generated by $\left\{y_{n}\right\}_{n<\omega}$. Suppose that $r_{1} y_{1}+\cdots+r_{n} y_{n}=0$ where $r_{i} \in R$. Then

$$
r_{1} c^{(2)}+r_{2} c^{(3)}+\cdots+r_{n} c^{(n+1)}=-r_{1} e_{1}-r_{2} e_{2}-\cdots-r_{n} e_{n} \cdot
$$

Since the first coordinate of the left hand side is zero, it follows that $r_{1}=0$. A repetition of the preceding argument shows that $r_{1}=r_{2}=$ $\cdots=r_{n}=0$. This implies that $A$ is free with $\left\{y_{n}\right\}_{n<\omega}$ for a basis.

We observe from [1] that a left $R$-module is torsionless if and only if it can be embedded as a submodule of a direct product of copies of $R$. We shall call a left $R$-module $G \aleph_{1}$-separable provided $G$ is flat, torsionless and that each countably generated submodule of $G$ is contained in a countably generated direct summand of $G$ (this definition parallels the definition given by $L$. Fuchs [4] in the context of $\aleph_{1}$-free groups). We now prove the main result of this section. The proof is modeled after that of Hill's [5].

THEOREM 2.2. A ring $R$ is left perfect if and only if each $\aleph_{1}$-separable left $R$-module is a direct sum of countably generated modules. 
Proof. If $R$ is left perfect, then by Theorem 3.2 [2] any flat left module is projective. Since an $\boldsymbol{N}_{1}$-separable left module is flat, it follows from Kaplansky's theorem [6] that each $\boldsymbol{\aleph}_{1}$-separable left $R$-module is a direct sum of countably generated modules.

Now suppose that $R$ is not left perfect. This implies by Theorem $P$ [1] that $R$ has a strictly decreasing sequence

$$
a_{1} R \supset a_{1} a_{2} R \supset \cdots \supset a_{1} \cdots a_{n} R \supset \cdots
$$

of principal right ideals. Set $P^{*}=\Pi_{\alpha<\Omega} R e_{\alpha}$ where $R e_{\alpha} \cong R$ for each $\alpha<\Omega(\Omega$ denotes the first uncountable ordinal). We construct a left submodule $G$ of $P^{*}$ such that $G=\bigcup_{\alpha<\Omega} G_{\alpha}$ where $\left\{G_{\alpha}\right\}_{\alpha<\Omega}$ is a monotone increasing chain defined as follows: $G_{0}=0, G_{1}=R e_{1}$ and suppose that $G_{\alpha}$ has been defined for each $\alpha<\beta$ such that the following conditions hold:

(i) If $\alpha$ is a limit ordinal, $\alpha<\beta, G_{\alpha}=\bigcup_{r<\alpha} G_{\gamma}$.

(ii) If $\alpha-1$ and $\alpha-2$ exist, $G_{\alpha}=G_{\alpha-1} \oplus R e_{\alpha-1}$.

(iii) If $\alpha-1$ exists and is a limit, there is a monotone increasing sequence $\sigma_{\alpha}(n)$ of ordinals less than $\alpha-1$ such that $\sigma_{\alpha}(n)-2$ is defined for each $n$ and such that $\sigma_{\alpha}(n)$ converges to $\alpha-1$. Then $c_{\alpha}^{(m)}=$ $\sum_{i \geqq m}\left(a_{m} \cdots a_{i}\right) e_{\sigma_{\alpha}(i)}$ for $m=1,2, \cdots$ and $G_{\alpha}$ is generated by $G_{\alpha-1}$ and $\left\{c_{\alpha}^{(n)}\right\}_{n<\omega}$.

(iv) If $\rho_{\gamma+1}$ denotes the natural projection of $P^{*}$ onto $\Pi_{\lambda<\gamma} R e_{\lambda}$ and if $\gamma+1<\alpha<\beta$, then $\rho_{\gamma+1}\left(G_{\alpha}\right)=G_{\gamma+1}$.

(v) $G_{\alpha}$ is not a direct summand of $G_{\alpha+1}$ if $\alpha$ is a limit ordinal.

(vi) $G_{\alpha}$ is flat for $\alpha<\beta$.

If $\beta$ is a limit ordinal we set $G_{\beta}=\bigcup_{\alpha<\beta} G_{\alpha}$ and if both $\beta-1$ and $\beta-2$ exist we set $G_{\beta}=G_{\beta-1} \oplus R e_{\beta-1}$. It is straightforward in either of the above two cases to show that (i)-(vi) hold for the collection $\left[G_{\alpha}\right]_{\alpha<\beta}$. Now suppose that $\beta-1$ is a limit ordinal. Define $\sigma_{\beta}(n)$ and $c_{\beta}^{(m)}$ so that (iii) is satisfied and define $G_{\beta}$ to be the submodule generated by $G_{\beta-1}$ and $\left\{c_{\beta}^{(c)}\right\}_{n<\omega}$. Suppose that $\gamma+1<\beta$ and consider $\rho_{\gamma+1}\left(G_{\beta}\right)$. To show that (iv) is satisfied, it clearly suffices to show that $\rho_{\gamma+1}\left(c_{\beta}^{(m)}\right) \in G_{\gamma+1}$. But this is a direct consequence of the fact that $c_{\beta}^{(m)}=\sum_{i \geqq m}\left(a_{m} \cdots a_{i}\right) e_{\sigma_{\beta}(i)}$ and that $\sigma_{\beta}(i)>\gamma+1$ for all $i$ larger than some integer $i_{0}$. To see that (v) holds, let $\Lambda_{\beta}$ be the set of ordinals $\left\{\sigma_{\beta}(1), \sigma_{\beta}(2), \cdots\right\}$ and let $I_{\beta}$ be the ordinals less than $\beta$ that are not in $\Lambda_{\beta}$. Let $B=G_{\beta} \cap \Pi_{I_{\beta}} R e_{\lambda}$, $A=G_{\beta} \cap \Pi_{A_{\beta}} R e_{\lambda}$ and let $S$ denote the finite sequences in $\Pi_{I_{\beta}} R e_{\lambda}$. It is routine to show that $G_{\beta}=B \oplus A$ and that $G_{\beta-1}=B \oplus S$. We observe that (up to isomorphism) our $A$ and $S$ here are the same as the $A$ and $S$, respectively, in Lemma 2.1. It follows that $G_{\beta-1}$ is not a direct summand of $G_{\beta}$. We also see that $G_{\beta-1}$ is flat since $B$ is necessarily flat and since $A$ is free. Thus the collection $\left[G_{\alpha}\right]_{\alpha \leqq \beta}$ satisfies (i)-(vi) and hence we obtain $G=\bigcup_{\alpha<\Omega} G_{\alpha}$ where $\left\{G_{\alpha}\right\}_{\alpha<\Omega}$ satisfies (i)-(vi). Note that $G$ is torsionless since $G$ is a submodule of $P^{*} . G$ is flat 
from (vi) since a direct limit of flat modules is flat. Property (v) implies that $G$ is not a direct sum of countably generated modules. Finally, property (iv) guarantees that $\rho_{r+1}$, when restricted to $G$, is a projection of $G$ onto $G_{\gamma+1}$. Thus $G$ is $\boldsymbol{\aleph}_{1}$-separable.

From the above proof, we obtain the following corollary.

Corollary 2.3. A ring $R$ is left perfect if and only if each $\aleph_{1}$-separable left $R$-module is projective.

3. Some remarks on pure injective modules over artinian rings. An interesting consequence of our Lemma 2.1 is that the direct sum of $\boldsymbol{\gamma}_{0}$ copies of a ring $R$ (as a left $R$-module) is not a direct summand of the corresponding direct product of $\boldsymbol{\aleph}_{0}$ copies of $R$ if $R$ is not left perfect. In this section we wish to consider in part the question of when the direct sum of infinitely many copies of $R$ (as a left $R$-module) is a direct summand of the corresponding direct product of copies of $R$. More generally, we consider the problem of determining when projective modules are pure injective modules in the sense of Warfield [7]. For commutative Noetherian rings we obtain a complete answer to both of the above questions. A submodule $A$ of a left $R$-module $B$ is called a pure submodule provided, for any right module $M$, the natural homomorphism $M \otimes A \rightarrow M \otimes B$ is injective. A module $Q$ is called pure injective, if for every module $B$ and pure submodule $A$, each homomorphism of $A$ into $Q$ extends to a homomorphism of $B$ into $Q$. Hence, if a pure injective module $Q$ is a pure submodule of a module $B$, then $Q$ is a direct summand of $B$. Our main theorems of this section follow the next lemma.

Lemma 3.1. If $R$ is a left artinian ring, then any pure submodule of a left projective $R$-module is a direct summand.

Proof. Suppose that $A$ is a pure submodule of a left projective module $P$ and suppose that $M$ is an arbitrary right $R$-module. From the exact sequence

$$
0=\operatorname{Tor}_{1}^{R}(M, P) \rightarrow \operatorname{Tor}_{1}^{R}(M, P / A) \rightarrow M \otimes A \rightarrow M \otimes P,
$$

we obtain that $\operatorname{Tor}_{1}^{R}(M, P / A)=0$ since the homomorphism $M \otimes A \rightarrow M \otimes P$ is injective. Hence $P / A$ is a flat left $R$-module. By Theorem $P[1]$, $P / A$ is projective and thus $A$ is a direct summand of $P$.

In what follows, $\sum A_{i}$ will denote the finitely nonzero vectors in the direct product $\Pi A_{i}$. 
THEOREM 3.2. If $R$ is a commutative artinian ring, then each projective $R$-module is pure injective. Moreover, if $R$ is a commutative Noetherian ring and if each projective $R$-module is pure injective, then $R$ is artinian.

Proof. First suppose that $R$ is a commutative artinian ring. It suffices to show that each free $R$-module is pure projective. By Proposition 9 [7], $R$ is pure injective as a module over itself. Let $F=$ $\sum_{\alpha} R$ be an arbitrary free $R$-module and let $P$ denote the direct product $P=\Pi_{\alpha} R$ containing $F$. It is elementary to see that $F$ is a pure submodule of $P$ and that $P$ is pure injective since $R$ is pure injective. By Theorem 3.4 [2], $P$ is also a projective $R$-module. Hence, by Lemma 3.1, $F$ is a direct summand of $P$ and therefore is pure injective.

Now suppose that $R$ is a commutative Noetherian ring for which each projective module is pure injective. Let $S$ and $A$ be as in Lemma 2.1. Note that $S=\sum_{\aleph_{0}} R$ and that $S \subseteq A \subseteq \Pi_{\aleph_{0}} R$. Therefore $S$ is pure in $A$ and is therefore a direct summand of $A$. Hence Lemma 2.1 yields that $R$ is a perfect ring. Since $R$ is also Noetherian, we have that $R$ is artinian.

COROLLARY 3.3. If $R$ is a commutative artinian ring, then the direct sum $\sum_{\alpha} R$ is a direct summand of the direct product $\Pi_{\alpha} R$ for each cardinal number $\alpha$. Moreover, if $R$ is a commutative Noetherian ring and if $\sum_{\aleph_{0}} R$ is a direct summand of $\Pi_{\aleph_{0}} R$, then $R$ is artinian.

We conclude our consideration of pure injective modules with an answer to the converse problem answered in Theorem 3.2, that is, we classify those rings for which every pure injective $R$-module is projective. Our solution here needs no initial assumptions on the ring.

THEOREM 3.4. A ring $R$ has the property that each pure injective left $R$-module is projective if and only if $R$ is semi-simple and artinian.

Proof. The sufficiency is clear. Hence suppose that $R$ has the property that each pure injective left $R$-module is projective. Since each injective left module is pure injective, it follows that each injective left $R$-module is also projective. By Theorem 5.3 [3] of Faith and Walker, we have that $R$ is quasi-Frobenius. Since each left $R$ module can be embedded as a pure submodule of a pure injective left $R$-module by Corollary 6 [7], we have that any left $R$-module is isomorphic to a pure submodule of a projective module. Since a quasiFrobenius ring is left artinian, it follows by Lemma 3.1 that each 
left $R$-module is projective. It is well-known that such a ring is a semi-simple artinian ring.

\section{REFERENCES}

1. H. Bass, Finitistic homological dimension and a homological generalization of semiprimary rings, Trans. Amer. Math. Soc. 95 (1960), 466-488.

2. S. U. Chase, Direct products of modules, Trans. Amer. Math. Soc. 97 (1960), 457473.

3. C. Faith and E. A. Walker, Direct sum representations of injective modules, J. of Algebra 5 (1967), 203-221.

4. L. Fuchs, Abelian groups, Hungarian Academy of Sciences, Budapest, 1958.

5. P. Hill, On the decomposition of groups (to appear).

6. I. Kaplansky, Projective modules, Ann. of Math. 68 (1958), 372-377.

7. R. B. Warfield, Purity and algebraic compactness for modules (to appear).

Received April 29, 1968. This work was supported in part by NASA Grant NGR44-005-037.

UNIVERSITY OF HOUSTON

Houston, TEXas 


\section{PACIFIC JOURNAL OF MATHEMATICS}

\section{EDITORS}

H. ROYDEN
Stanford University
Stanford, California

\section{R. R. PHELPS}

University of Washington

Seattle, Washington 98105
J. DUGUNDJI

Department of Mathematics

University of Southern California

Los Angeles, California 90007

\section{RICHARD ARENS}

University of California

Los Angeles, California 90024

\section{ASSOCIATE EDITORS}

E. F. BeCKenbaCh
B. H. NEUMANN

F. WOLF
K. YoshidA

\section{SUPPORTING INSTITUTIONS}

\author{
UNIVERSITY OF BRITISH COLUMBIA \\ CALIFORNIA INSTITUTE OF TECHNOLOGY \\ UNIVERSITY OF CALIFORNIA \\ MONTANA STATE UNIVERSITY \\ UNIVERSITY OF NEVADA \\ NEW MEXICO STATE UNIVERSITY \\ OREGON STATE UNIVERSITY \\ UNIVERSITY OF OREGON \\ OSAKA UNIVERSITY \\ UNIVERSITY OF SOUTHERN CALIFORNIA
}

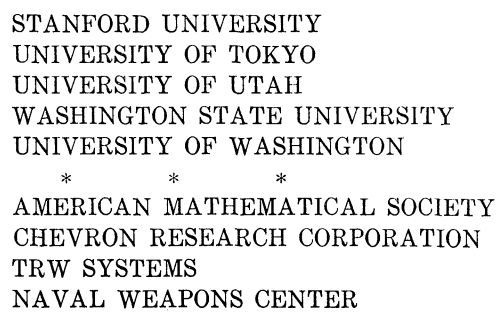

The Supporting Institutions listed above contribute to the cost of publication of this Journal, but they are not owners or publishers and have no responsibility for its content or policies.

Mathematical papers intended for publication in the Pacific Journal of Mathematics should be in typed form or offset-reproduced, double spaced with large margins. Underline Greek letters in red, German in green, and script in blue. The first paragraph or two must be capable of being used separately as a synopsis of the entire paper. It should not contain references to the bibliography. Manuscripts, in duplicate if possible, may be sent to any one of the four editors. Please classify according to the scheme of Math. Rev. 36, 1539-1546. All other communications to the editors should be addressed to the managing editor, Richard Arens, University of California, Los Angeles, California, 90024.

50 reprints are provided free for each article; additional copies may be obtained at cost in multiples of 50 .

The Pacific Journal of Mathematics is published monthly. Effective with Volume 16 the price per volume (3 numbers) is $\$ 8.00$; single issues, $\$ 3.00$. Special price for current issues to individual faculty members of supporting institutions and to individual members of the American Mathematical Society: $\$ 4.00$ per volume; single issues $\$ 1.50$. Back numbers are available.

Subscriptions, orders for back numbers, and changes of address should be sent to Pacific Journal of Mathematics, 103 Highland Boulevard, Berkeley, California, 94708.

PUBLISHED BY PACIFIC JOURNAL OF MATHEMATICS, A NON-PROFIT CORPORATION

Printed at Kokusai Bunken Insatsusha (International Academic Printing Co., Ltd.), 7-17, Fujimi 2-chome, Chiyoda-ku, Tokyo, Japan. 


\section{Pacific Journal of Mathematics \\ Vol. 29, No. $2 \quad$ June, 1969}

Bruce Langworthy Chalmers, On boundary behavior of the Bergman kernel function and related domain functionals ................... 243

William Eugene Coppage, Peirce decomposition in simple Lie-admissible power-associative rings .............................. 251

Edwin Duda, Compactness of mappings...................... 259

Earl F. Ecklund Jr., On prime divisors of the binomial coefficient......... 267

Don E. Edmondson, A modular topological lattice ............... 271

Phillip Alan Griffith, A note on a theorem of Hill ................... 279

Marcel Herzog, On finite groups with independent cyclic Sylow

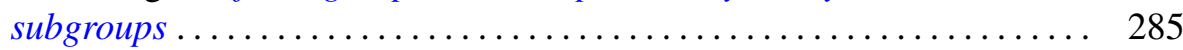

James A. Huckaba, Extensions of pseudo-valuations................. 295

S. A. Huq, Semivarieties and subfunctors of the identity functor ........ 303

I. Martin (Irving) Isaacs and Donald Steven Passman, Finite groups with small character degrees and large prime divisors. II ............ 311

Carl Kallina, A Green's function approach to perturbations of periodic

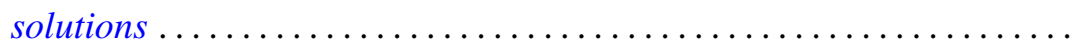

$\mathrm{Al}$ (Allen Frederick) Kelley, Jr., Analytic two-dimensional subcenter manifolds for systems with an integral ....................

Alistair H. Lachlan, Initial segments of one-one degrees ............ 351

Marion-Josephine Lim, Rank k Grassmann products ............. 367

Raymond J. McGivney and William Henry Ruckle, Multiplier algebras of

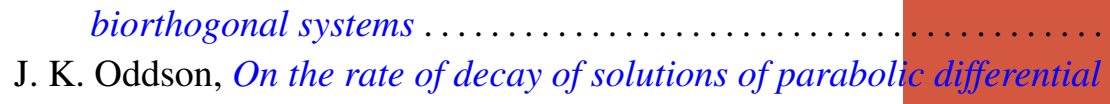

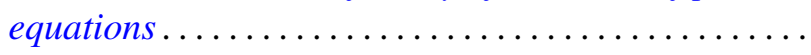

Helmut R. Salzmann, Geometries on surfaces ........... .

Annemarie Schlette, Artinian, almost abelian groups and their groups of automorphisms ............................

Edgar Lee Stout, Additional results on modules over polydisc algebras ...

Lajos Tamássy, A characteristic property of the sphere . .

Mark Lawrence Teply, Some aspects of Goldie's torsion theory. ...

Freddie Eugene Tidmore, Extremal structure of star-shaped sets ...

461

Leon Jarome Weill, Unconditional and shrinking bases in locally convex spaces... 